\title{
Comparison of functional activation responses from the auditory cortex derived using multi-distance frequency domain and continuous wave near-infrared spectroscopy
}

\author{
Penaz Parveen Sultana Mohammad, ${ }^{a}$ Sittiprapa Isarangura $\odot$, \\ Ann Eddins $\odot$, , and Ashwin B. Parthasarathy $\oplus^{a, *}$ \\ ${ }^{a}$ University of South Florida, Department of Electrical Engineering, Tampa, Florida, \\ United States \\ ${ }^{b}$ University of South Florida, Department of Communication Sciences and Disorders, Tampa, \\ Florida, United States
}

\begin{abstract}
Significance: Quantitative measurements of cerebral hemodynamic changes due to functional activation are widely accomplished with commercial continuous wave (CW-NIRS) instruments despite the availability of the more rigorous multi-distance frequency domain (FD-NIRS) approach. A direct comparison of the two approaches to functional near-infrared spectroscopy can help in the interpretation of optical data and guide implementations of diffuse optical instruments for measuring functional activation.

Aim: We explore the differences between CW-NIRS and multi-distance FD-NIRS by comparing measurements of functional activation in the human auditory cortex.

Approach: Functional activation of the human auditory cortex was measured using a commercial frequency domain near-infrared spectroscopy instrument for $70 \mathrm{~dB}$ sound pressure level broadband noise and pure tone $(1000 \mathrm{~Hz})$ stimuli. Changes in tissue oxygenation were calculated using the modified Beer-Lambert law (CW-NIRS approach) and the photon diffusion equation (FD-NIRS approach).
\end{abstract}

Results: Changes in oxygenated hemoglobin measured with the multi-distance FD-NIRS approach were about twice as large as those measured with the CW-NIRS approach. A finiteelement simulation of the functional activation problem was performed to demonstrate that tissue oxygenation changes measured with the CW-NIRS approach is more accurate than that with multi-distance FD-NIRS.

Conclusions: Multi-distance FD-NIRS approaches tend to overestimate functional activation effects, in part due to partial volume effects.

(C) The Authors. Published by SPIE under a Creative Commons Attribution 4.0 International License. Distribution or reproduction of this work in whole or in part requires full attribution of the original publication, including its DOI. [DOI: 10.1117/1.NPh.8.4.045004]

Keywords: near-infrared spectroscopy (NIRS); frequency domain measurements; continuous wave-NIRS; functional activation; functional near-infrared spectroscopy; auditory stimulation.

Paper 21017RRR received Apr. 14, 2021; accepted for publication Nov. 29, 2021; published online Dec. 15, 2021.

\section{Introduction}

Over the last decade, functional near-infrared spectroscopy (fNIRS) has emerged as a reliable non-invasive method for monitoring cortical activity in the brain. ${ }^{1-3}$ fNIRS has been used for a variety of applications such as to study neuronal activation of brain circuits, ${ }^{4-6}$ to image functional/resting state connectivity of the human brain, ${ }^{4,7,8}$ to examine and characterize cognitive

*Address all correspondence to Ashwin B. Parthasarathy, ashwinbp@usf.edu 
behavior, ${ }^{9-11}$ and to study/implement brain-computer interfaces. ${ }^{12-14}$ fNIRS instruments are especially useful for characterizing functional hemodynamic changes associated with the auditory system. It is generally difficult to measure cerebral activity in response to activations of the auditory cortex with clinical imaging modalities such as x-ray computed tomography or magnetic resonance imaging because instrument sounds increase background noise, which could corrupt the careful presentation of auditory stimuli to subjects and thereby significantly bias the results of an experiment. In part, because of these advantages, several recent studies ${ }^{7,15-17}$ have used commercial fNIRS instruments to characterize functional stimulation of auditory cortex in humans. For example, Chen et al. ${ }^{7}$ measured the hemodynamic responses from the auditory cortex for 440 and $554 \mathrm{~Hz}$ pure tones and a $1000-\mathrm{Hz}$ frequency modulated or warble tone. Hong and Santosa ${ }^{16}$ performed a similar experiment to study hemodynamic responses for "natural" sound stimuli, such as English and non-English words, annoying sounds, and nature sounds. Issa et al. ${ }^{18}$ measured the hemodynamic changes in the auditory cortex when presented with a pure tone stimulus of 750 and $8000 \mathrm{~Hz}$ as well as broadband noise. The primary goal of these experiments was to measure or image focal, i.e., localized, changes in cerebral tissue oxygenation within the auditory cortex - this could be thought of as the fundamental question of fNIRS experiments. The primary auditory cortex in humans spans $\sim 1650 \mathrm{~mm}^{3}$ within Heschl's gyrus of the temporal lobe and is organized along multiple functional dimensions, the most prominent one being tonotopic. ${ }^{19,20} \mathrm{We}$, therefore, expect that pure tone stimuli will activate a more focal region of the auditory cortex, whereas broadband noise will activate a broader region. ${ }^{19,21,22}$

Traditionally, fNIRS has been implemented using a continuous wave approach [continuous wave near-infrared spectroscopy (CW-NIRS)] because it offers a fast and convenient way to measure focal changes in oxygenated hemoglobin $\left(\Delta C_{\mathrm{HbO}}\right)$ and deoxygenated hemoglobin $\left(\Delta C_{\mathrm{HbR}}\right)$. Indeed, most commercial implementations of NIRS follow this approach. In CWNIRS, tissue is illuminated with a light source of constant intensity, and the attenuated light emitted from the surface of tissue, measured 1 to $2.5 \mathrm{~cm}$ away on the tissue surface, is used to calculate the absorbance or optical density. Changes in the optical density, e.g., due to neuronal activation, are converted to relative changes in the concentration of tissue chromophores using the modified Beer-Lambert (MBL) law. ${ }^{1}$ The MBL approach implements a simple correction factor (differential path length factor) to account for the extension of light pathlength due to multiple scattering in tissue. ${ }^{23} \mathrm{~A}$ more quantitative alternative to CW-NIRS is frequency domain NIRS (FD-NIRS), which estimates absolute concentrations of oxygenated and deoxygenated hemoglobin from measurements that can be directly fit to solutions to the photon diffusion equation. ${ }^{24}$ In its most common implementation, FD-NIRS measures the intensity and phase shift of backscattered light from multiple source detection separations, when tissue is illuminated by an intensity modulated source at different wavelengths. The intensity modulated source sets up a diffusive photon density wave in tissue, the wave vector of which is used to estimate optical tissue properties. Since this approach directly estimates scattering and absorption coefficients of tissue, it holds the potential to measure baseline concentrations of oxygenated and deoxygenated hemoglobin. However, despite being a more quantitative alternative and the availability of commercial FD-NIRS instruments (e.g., OxiplexTS, Imagent from ISS Inc.), studies that use FD-NIRS have primarily focused on global changes in concentrations of $C_{\mathrm{HbO}}$ and $C_{\mathrm{HbR}}$ such as hypoxia and cerebral ischemia, ${ }^{15,25-28}$ and the literature that discusses the implementation of this more quantitative technique to measure the focal changes in $C_{\mathrm{HbO}}$ and $C_{\mathrm{HbR}}$ such as those involving functional activation experiments is scant.

In this study, we explored this apparent discrepancy and compared typical implementations of CW- and FD-NIRS instruments to measure functional activation. Specifically, we characterized the hemodynamic response of the human auditory cortex due to functional activation using a commercial FD-NIRS instrument and analyzed the results using both CW and multi-distance FD approaches. Using in vivo data from functional activation of healthy human auditory cortex, we demonstrate that hemodynamic responses estimated from CW- and multi-distance FD-NIRS approaches differ significantly even though they are measured at the same time and from the same physical position. Furthermore, using finite-element simulations (NIRFAST ${ }^{29}$ ), we suggest that FD-NIRS estimates of cerebral oxygenation measured using multi-distance methods tend to overestimate focal hemodynamic changes, in part due to the partial volume effect, when compared with single-distance CW approaches. 


\section{Experimental Methods}

\subsection{Frequency Domain Near-Infrared Spectroscopy Instrument}

Cerebral hemodynamic changes due to functional activation were measured using a commercial frequency-domain near-infrared spectroscopy instrument (Imagent, ISS Inc., IL, USA). The instrument features eight laser diodes as light sources at two different wavelengths (690 and $830 \mathrm{~nm}$ ) and eight photomultiplier tubes (A through $\mathrm{H}$ ) for use as detectors. The intensity of the lasers was modulated at $110 \mathrm{MHz}$. The instrument demodulates the photon fluence measured at each detector to estimate AC, DC, and phase of the detected photon density wave. ${ }^{30}$ The laser diodes were time multiplexed to illuminate at one source position/wavelength (1 through 4) at a time, whereas photon fluence is measured at all detectors simultaneously, resulting in an effective total frame rate of $10 \mathrm{~Hz}$. The system was warmed up for $10 \mathrm{~min}$ prior to the start of the measurement. Light from the sources was directed to source positions in a custom optical probe (see Sec. 2.2) using a bifurcated fiber-optic cable (multi-mode bundle, 1-mm diameter). Light detected from eight detector positions was directed to photomultiplier tubes A through $\mathrm{H}$ using 3-mm-diameter fiber bundles. Thus AC, DC, and phase measurements at each detector facilitated multi-distance frequency domain NIRS estimations of baseline tissue optical properties. The average source-detector separation was $\sim 3 \mathrm{~cm}$. Table 1 lists the source-detector separations (in $\mathrm{cm}$ ) for each detection and source position.

\subsection{Probe Design and Placement}

A custom optical probe was 3D-printed to hold source/detector fibers at their required separations. A schematic for the arrangement for source and detector fibers (four source positions and eight detector positions) is shown in Fig. 1. Large circles highlight the locations of the detector fibers, and the smaller circles highlight the locations of the source fibers. Using the international 10-20 system as a guide, the probe was placed such that center of the sources was on either side of the T4 position or over the right auditory cortex ${ }^{7,16,18,31,32}$ as shown in Fig. 2. The T4 position was identified on each subject from individual measurements of head circumference, inionnasion, and tragus-tragus distances. The probe was held in place using two thin Velcro straps on both sides. A USB otoscope was used to minimize the effect of hair at the source and detector positions after the placement of the probe. The scope was used to check and move hair from obstructing the fibers. The intensity values were checked after the placement of the probe to ensure a good signal response.

Table 1 Distance $(\mathrm{cm})$ between the sources and detectors for fiberoptic probe shown in Fig. 1.

\begin{tabular}{lcccc}
\hline \hline & Source 1 & Source 2 & Source 3 & Source 4 \\
\hline Detector A & 2.2 & 2.9 & 3.8 & 4.6 \\
Detector B & 1.6 & 2.1 & 2.9 & 3.6 \\
Detector C & 3.6 & 2.9 & 2.1 & 1.6 \\
Detector D & 4.6 & 3.8 & 2.9 & 2.2 \\
Detector E & 2.2 & 2.9 & 3.8 & 4.6 \\
Detector F & 1.6 & 2.1 & 2.9 & 3.6 \\
Detector G & 3.6 & 2.9 & 2.1 & 1.6 \\
Detector H & 4.6 & 3.8 & 2.9 & 2.2 \\
\hline \hline
\end{tabular}




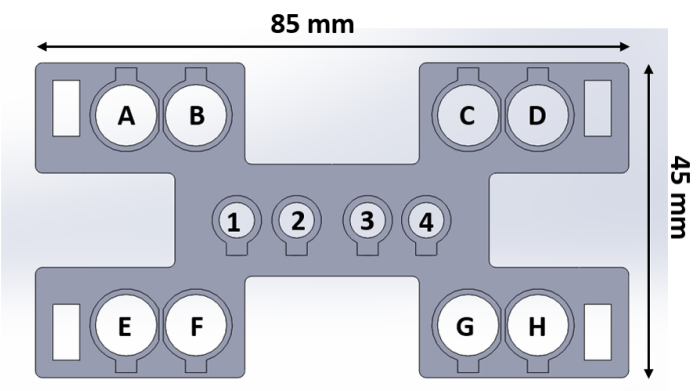

Fig. 1 Schematic of custom fiberoptic probe with four source positions and eight detector positions. Source positions are denoted 1 through 4 , and detector positions are denoted $A$ to $H$. The corresponding source-detector separations are listed in Table 1.

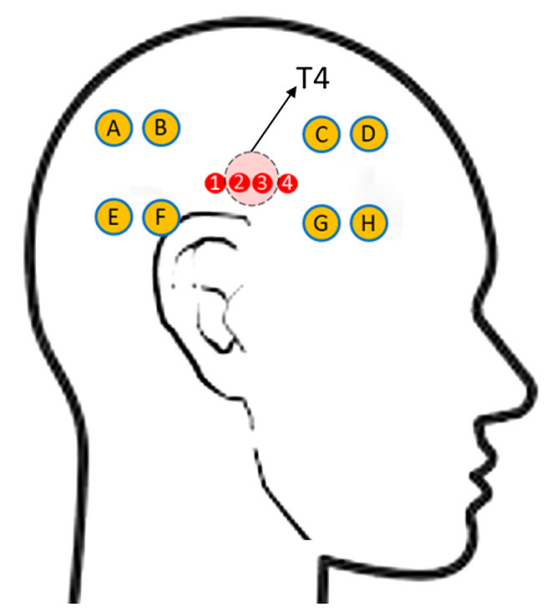

Fig. 2 Schematic representing positioning of sources and detectors on the scalp over the auditory cortex. Red circles represent the source positions, the center of which is placed above the T4 position over the right ear. Yellow circles represent detector positons, with detectors A, B, E, and $\mathrm{F}$ sampling posterior auditory cortex and detectors $\mathrm{C}, \mathrm{D}, \mathrm{G}$, and $\mathrm{H}$ sampling the anterior auditory cortex.

\subsection{Subjects}

Nine healthy adults (six female and three male), median age of 29 years (range: 20 to 35 years old), with normal hearing ( $\leq 25 \mathrm{~dB}$ HL, 250 to $8000 \mathrm{~Hz}$ ), participated in this study. All procedures were approved by USF's Institutional Review Board, and subjects provided written informed consent prior to participation.

\subsection{Experimental Protocol}

Functional activation measurements were performed in a double-walled, sound-treated booth with lights dimmed to limit the effect of ambient lights on the measurements. Responses to two different auditory stimuli were evaluated, $1000 \mathrm{~Hz}$ pure tone and broadband noise; the order of presentation was randomized across subjects. The stimuli were generated digitally [RP2, Tucker Davis Technologies (TDT), Alachua, FL], routed to a programmable attenuator (PA5, TDT), headphone buffer (HB6, TDT), and presented binaurally at $70 \mathrm{~dB}$ SPL via insert earphones (ER-2, Etymotic Research Inc., Elk Grove, IL). Stimuli were controlled using SigGen and BioSig software (TDT) with each stimulus having a duration of $10 \mathrm{~s}$, a 30-s interstimulus inter$\mathrm{val}$, and 21 repetitions. The experiment protocol is similar to those in publications that have used fNIRS to study the auditory response. ${ }^{16,33,34}$ The overall recording time was $\sim 14$ min including a 
$60 \mathrm{~s}$ baseline period prior to stimuli presentation. NIRS measurements of average intensity (DC), amplitude (AC), and phase $(\phi)$ were continuously recorded along with trigger markers to indicate the start and end of the stimulus presentation. Data were postprocessed to estimate hemodynamic changes due to functional activation.

\section{$2.5 C W-N I R S$ Analysis}

The MBL approach was used to calculate relative changes in concentrations of oxygenated hemoglobin $\left(C_{\mathrm{HbO}}\right)$ and deoxygenated hemoglobin $\left(C_{\mathrm{HbR}}\right)$ due to functional activation, with custom MATLAB scripts (Mathworks, Natick, MA). To reduce the impact of the room or other stray lights on the recorded data, we used the AC amplitude to perform the CW-NIRS analysis. ${ }^{35}$ The recorded amplitude at each source-detector separation was first converted to optical density by normalizing the intensity time traces at each wavelength with the corresponding average intensity at baseline. A moving average window $(0.4 \mathrm{~s})$ was used to remove motion artifacts in the data ${ }^{31}$ the local variance in OD within the moving window was calculated, and regions/trials that exhibited large local standard deviation ( $>2$ times mean variance) were excluded from the analysis. Motion artifact corrected time courses were band pass filtered $(0.01$ to $0.5 \mathrm{~Hz})$ and split into $30 \mathrm{~s}$ epochs corresponding to each trial of auditory stimuli. For each trial, the change in optical density was computed by subtracting the baseline (i.e., prestimulation) optical density from the measured optical density. Finally, changes in optical density were then converted to changes in $C_{\mathrm{HbO}}$ and $C_{\mathrm{HbR}}$ on a trial-by-trial basis by inverting the following equation:

$$
\left[\begin{array}{c}
\Delta \mathrm{OD}\left(\lambda_{1}\right) \\
\Delta \mathrm{OD}\left(\lambda_{2}\right)
\end{array}\right]=\left[\begin{array}{ll}
\epsilon_{\mathrm{Hbo}}\left(\lambda_{1}\right) \operatorname{DPF}\left(\lambda_{1}\right) \rho & \epsilon_{\mathrm{HbR}}\left(\lambda_{1}\right) \operatorname{DPF}\left(\lambda_{1}\right) \rho \\
\epsilon_{\mathrm{Hbo}}\left(\lambda_{2}\right) \operatorname{DPF}\left(\lambda_{2}\right) \rho & \epsilon_{\mathrm{HbR}}\left(\lambda_{2}\right) \operatorname{DPF}\left(\lambda_{2}\right) \rho
\end{array}\right]\left[\begin{array}{c}
\Delta C_{\mathrm{HbO}}\left(\lambda_{1}\right) \\
\Delta C_{\mathrm{HbR}}\left(\lambda_{2}\right)
\end{array}\right] .
$$

Here DPF is the differential pathlength factor; DPF is detector specific for each subject and was calculated as $\operatorname{DPF}(\lambda)=\frac{\sqrt{3 \mu_{s}^{\prime}(\lambda)}}{2 \sqrt{\mu_{a}(\lambda)}}$, where $\mu_{a}$ and $\mu_{s}^{\prime}$ were estimated from the baseline data calculated with FD-NIRS analysis, ${ }^{27} \rho$ is the source-detector separation in $\mathrm{cm}$, and $\epsilon_{\mathrm{HbO} / \mathrm{HbR}}\left(\lambda_{i}\right)$ is the molar extinction coefficient of $\mathrm{HbO} / \mathrm{HbR}$ at wavelength $\lambda_{i}$ in $\mathrm{cm}^{-1} \mathrm{~mol}^{-1} \cdot{ }^{36,37} \mathrm{CW}-\mathrm{NIRS}$ data are especially susceptible to instrumental drifts and coupling errors. Hence, the hemodynamic responses were detrended to remove gradual drifts in the data. Detrending was performed on a trial-by-trial basis to reduce the effects of any long-term data drifts. Results from multiple trials and from all subjects were averaged. Figures S1 and S2 in the Supplementary Material show the CW-NIRS data processing pipeline for an exemplar subject.

\subsection{Multi-Distance Frequency Domain NIRS Analysis}

Estimates of absolute concentrations of oxygenated and deoxygenated hemoglobin were computed for the functional activation measurements using the multi-distance frequency domain approach. ${ }^{30,38}$ Intensity and phase data were corrected for motion artifacts, as described earlier. The corrected data were filtered using a low-pass filter with a cutoff frequency of $0.1 \mathrm{~Hz}$. A phantom calibration procedure was employed to estimate and correct for probe-tissue coupling coefficients. ${ }^{39,40}$ At each time point, calibrated and filtered amplitudes $(A)$ measured at each detector were linearized $\left(\log \left(\rho^{2} A(\rho)\right)\right)$ and plotted as a function of source-detector separation $(\rho)$; a linear fit was performed to estimate the slope $k_{r}$. Similarly, a linear fit to phase $(\phi)$ as a function of $\rho$ was performed to estimate the slope $k_{i}$. Tissue optical properties $\mu_{a}$ and $\mu_{s}^{\prime}$ were estimated from the following expressions: ${ }^{40}$

$$
\mu_{a}(\lambda)=\frac{\omega}{2 v}\left(\frac{\kappa_{r}(\lambda)}{\kappa_{i}(\lambda)}-\frac{\kappa_{i}(\lambda)}{\kappa_{r}(\lambda)}\right), \quad \mu_{s}^{\prime}(\lambda)=\frac{2 v}{3 \omega}\left(-\kappa_{r}(\lambda) \kappa_{i}(\lambda)\right),
$$

where $\omega=2 \pi f_{\text {mod }}, f_{\text {mod }}$ is the modulation frequency and $v$ is the speed of light in the tissue. The measured absorption coefficient was corrected for water absorption $\left(\mu_{a}^{830}=0.029 \mathrm{~cm}^{-1}\right.$ and $\mu_{a}^{690}=0.0049 \mathrm{~cm}^{-1}$ ), assuming that water accounts for $75 \%$ of brain matter, ${ }^{41,42-44}$ and 
converted to time courses of concentrations of oxygenated $\left(C_{\mathrm{HbO}}\right)$ and deoxygenated hemoglobin $\left(C_{\mathrm{HbR}}\right)$. In a manner similar to CW-NIRS analysis, the time courses of $C_{\mathrm{HbO}}$ and $C_{\mathrm{HbR}}$ were split into epochs corresponding to auditory stimuli. Changes in concentrations of $C_{\mathrm{HbO}}$ and $C_{\mathrm{HbR}}$ were computed by subtracting the baseline (i.e., prestimulus) concentration from the respective time courses of $C_{\mathrm{HbO}}$ and $C_{\mathrm{HbR}}$. Results from multiple trials and from all subjects were averaged. Note that some detectors feature FD-NIRS measurements at a source-detector separation of $1.6 \mathrm{~cm}$. Since extracerebral contributions are likely to be significant at these separations, amplitude and phase data collected at this short separation were excluded from FD-NIRS analysis. Figures S3 and S4 in the Supplementary Material show the FD-NIRS data processing pipeline for an exemplar subject.

\subsection{Statistical Analysis of Functional Activation Responses}

The average changes in oxygenated and deoxygenated hemoglobin during the activation period were calculated to quantitatively characterize the functional activation responses. Briefly, the highest point (i.e., maximum change) in the hemodynamic response within the activation period was identified. The average change in concentrations $1.5 \mathrm{~s}$ before and after the time of maximum response was computed and used as a quantitative metric of functional activation. The Wilcoxon signed rank test was performed to test if the average hemodynamic change was statistically greater than zero. In addition, the subject-wise paired Wilcoxon signed rank test was performed to test if the CW-NIRS responses at each source-detector separation were smaller from the corresponding FD-NIRS responses. All statistical testing was performed at 5\% significance level. The statistical analyses performed in this paper are focused on the oxygenated hemoglobin because signals from the cortex are influenced by oxygenated hemoglobin changes to a greater extent $(76 \%)$ and oxygenated hemoglobin responses were more robust to noise. ${ }^{45,46}$

\section{Results}

\subsection{CW-NIRS and FD-NIRS Estimates of Hemodynamic Changes due to Functional Activation}

We first highlight the baseline concentrations of oxygenated and deoxygenated hemoglobin at each detector for all subjects (Table 2). Baseline concentrations were obtained by multi-distance FD-NIRS analysis of phase and amplitude. Note that the source-detector separations (Table 1) vary for each detector (per probe geometry in Fig. 1). Therefore, the results in Table 2 represent an ensemble sampling of the auditory cortex regions both laterally and in depth. FD-NIRS analysis was possible on data from only six subjects. One subject's data was unusable due to errors

Table 2 Baseline concentrations of oxygenated and deoxygenated hemoglobin $(\mu \mathrm{M})$ averaged across all subjects for detectors $\mathrm{A}$ to $\mathrm{H}$.

\begin{tabular}{lcc}
\hline \hline & $C_{\mathrm{HbO}}(\mu \mathrm{M})$ & $C_{\mathrm{HbR}}(\mu \mathrm{M})$ \\
\hline Detector A & $70.7 \pm 11.7$ & $26.8 \pm 4.07$ \\
Detector B & $69.0 \pm 13.4$ & $33.1 \pm 8.9$ \\
Detector C & $42.0 \pm 16.2$ & $17.9 \pm 3.1$ \\
Detector D & $48.0 \pm 12.6$ & $20.5 \pm 3.2$ \\
Detector E & $58.9 \pm 10.2$ & $26.7 \pm 4.5$ \\
Detector F & $58.8 \pm 3.2$ & $28.1 \pm 2.5$ \\
Detector G & $39.5 \pm 13.5$ & $20.5 \pm 3.5$ \\
Detector H & $41.3 \pm 13.1$ & $20.1 \pm 2.5$ \\
\hline \hline
\end{tabular}



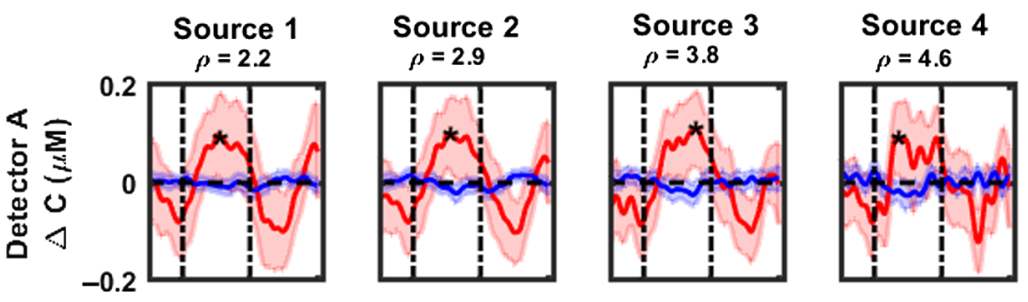

FD
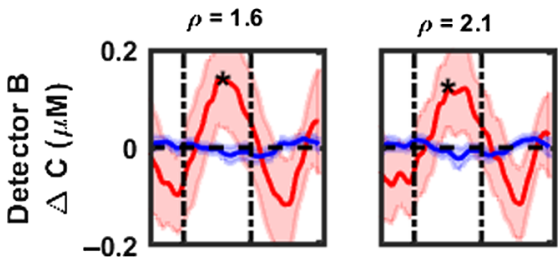

$\rho=2.9$
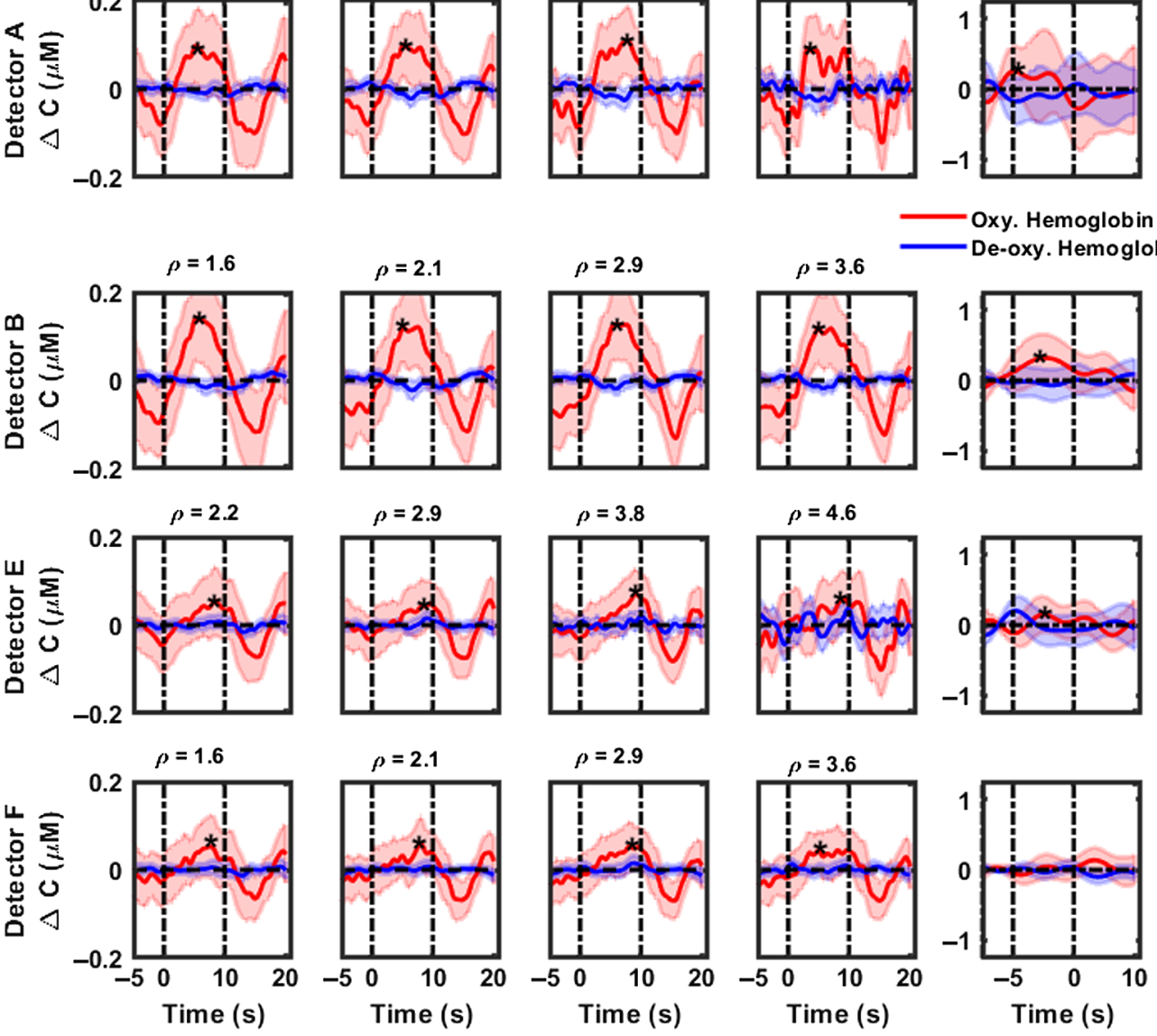

Fig. 3 CW-NIRS and FD-NIRS analyses of functional activation (1000 Hz pure tone) recorded over the posterior auditory cortex (detectors A, B, E, and F) from all subjects. Rows represent hemodynamic responses recorded at each detector. The first four columns highlight responses recoded at each source position using CW-NIRS (left to right, increasing source-detector separation) and FD-NIRS (last column). In each plot, changes in concentration of oxygenated hemoglobin are plotted in red, and the concentration of deoxyhemoglobin is plotted in blue. The title of each subplot denotes the source-detector separation in $\mathrm{cm}$ for that measurement channel. Note that the $y$ axis scale for FD-NIRS measurements is larger than that of CW-NIRS measurements. Dashed vertical lines indicate the start and end of the stimulus. Shaded regions indicate the variance in the responses. Note that source 1 is closest to and source 4 is farthest from all detectors. Plots marked with a "*” indicate statistically significant responses.

during the calibration process. Data from two other subjects were excluded because intermittent saturation effects during the experiment rendered the data unusable. CW-NIRS analysis utilizes the DPF calculated with FD-NIRS analysis; hence the data from six subjects were used.

Figures 3 and 4 highlight the cumulative hemodynamic response curves from CW-NIRS (first four columns) and FD-NIRS (last column) analyses of $1000 \mathrm{~Hz}$ pure tone activation, recorded over the posterior and anterior auditory cortices respectively, from six subjects. Changes in concentrations of oxygenated hemoglobin are displayed as solid red lines, with corresponding shaded regions denoting $95 \%$ confidence intervals. Similarly, changes in concentrations of deoxygenated hemoglobin and corresponding 95\% confidence intervals are highlighted in blue. The period between the dashed vertical lines, i.e., between 0 and $10 \mathrm{~s}$, represents the activation duration. With CW-NIRS analysis, a robust increase of 0.107 to $0.13 \mu \mathrm{M}$ is observed in oxygenated hemoglobin (maximum value within duration of stimulus presentation), primarily in the auditory cortex region superior to the T4 position (detectors A, B, and C). Hemodynamic responses were quantified as described in Sec. 2.7 (see also Table 3). Statistically significant 

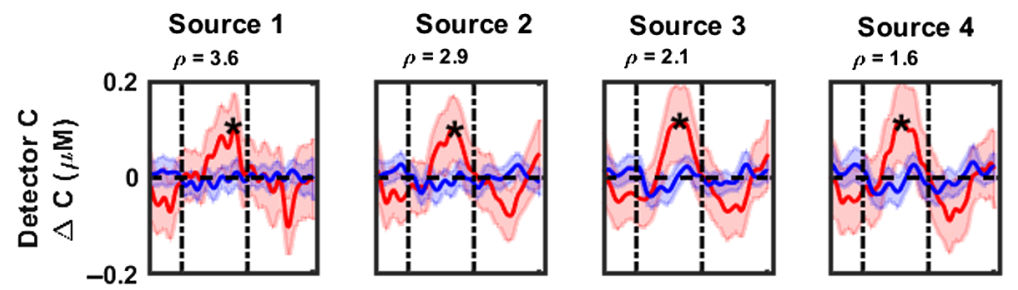

FD
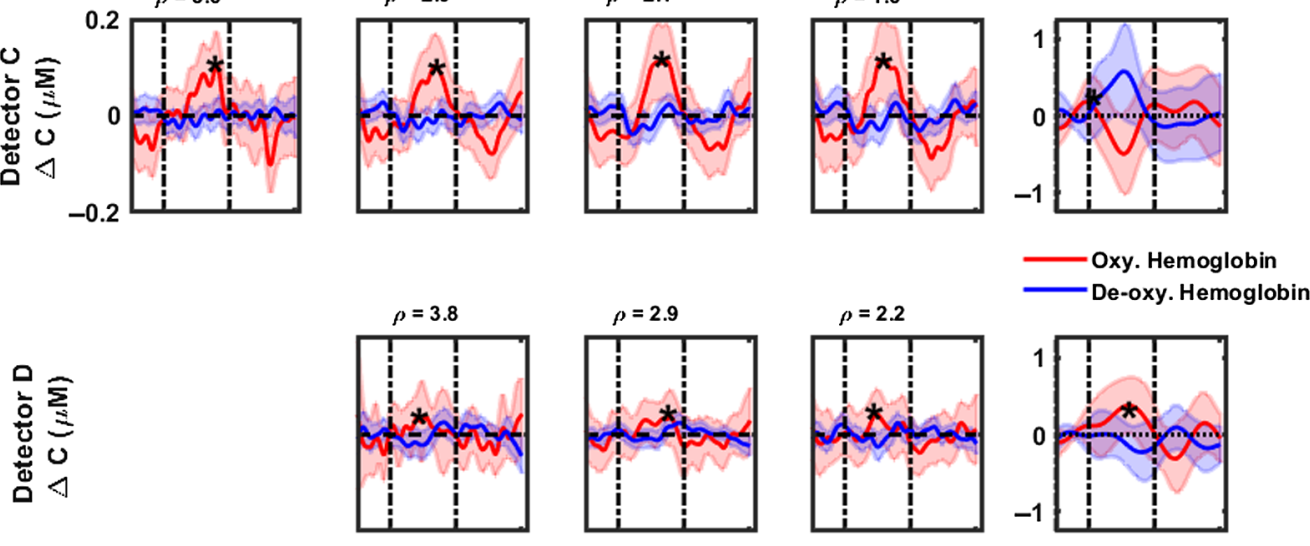

0
$\frac{1}{0}$
0
0
0
0
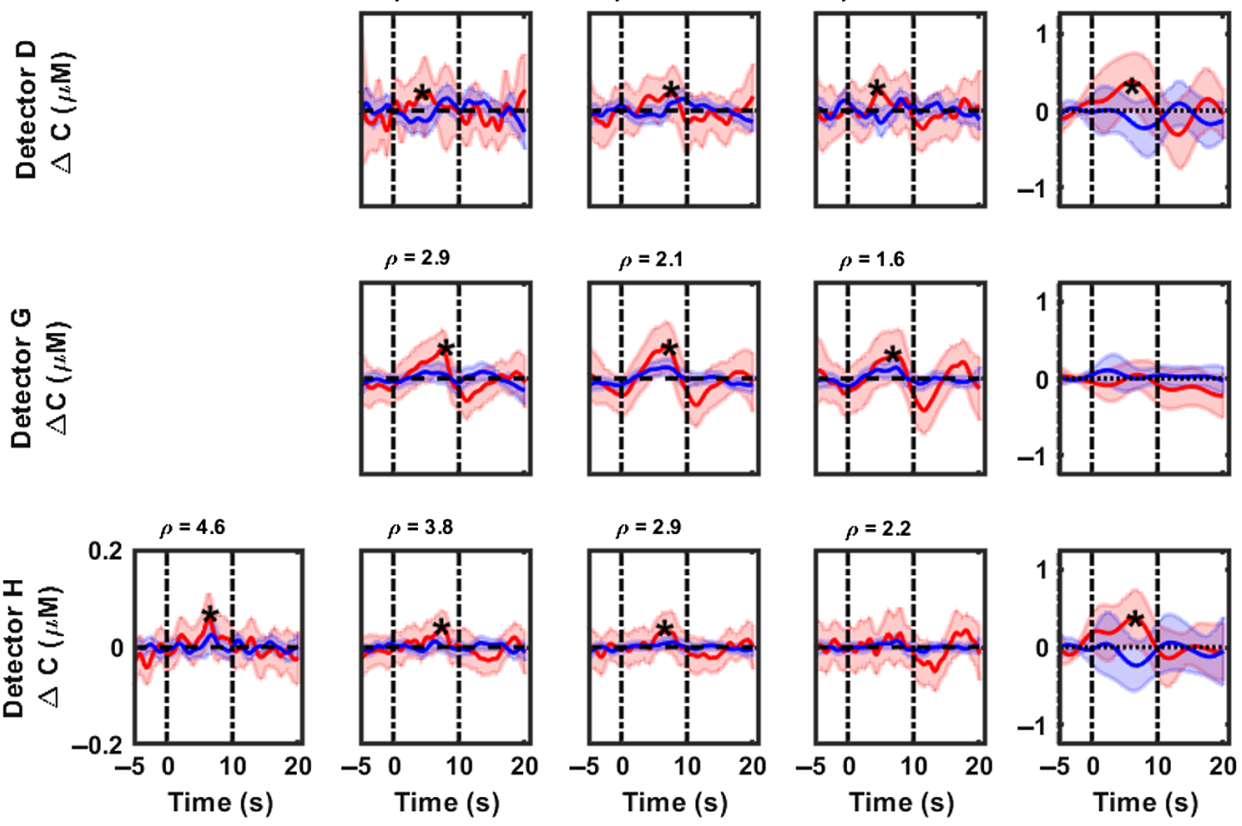

Fig. 4 CW-NIRS and FD-NIRS analyses of functional activation (1000 Hz pure tone) recorded over the anterior auditory cortex (detectors C, D, G, and H) from all subjects. Rows represent hemodynamic responses recorded at each detector. The first four columns highlight responses recoded at each source position using CW-NIRS (left to right, decreasing source-detector separation) and FD-NIRS (last column). In each plot, changes in concentration of oxygenated hemoglobin are plotted in red, and the concentration of deoxyhemoglobin is plotted in blue. The title of each subplot denotes the source-detector separation in $\mathrm{cm}$ for that measurement channel. Note that the $y$ axis scale for FD-NIRS measurements is larger than that of CW-NIRS measurements. Dashed vertical lines indicate the start and end of the stimulus. Shaded regions indicate the variance in the responses. Note that source 4 is closest to and source 1 is farthest from all detectors. Plots for source 1. Detector $D$ and $G$ are not included here because they were too noisy. Plots marked with a "*” indicate statistically significant responses.

responses (Wilcoxon signed rank test, testing for response $>0$ ) were observed in most sourcedetector pairs in the posterior auditory cortex (marked by a “*” in Fig. 3). Hemodynamic changes measured in the anterior auditory cortex (Fig. 4) were modest compared with those in the posterior auditory cortex, indicating the presence of the tonotopic response to pure tone stimuli. No significant changes were observed in the concentration of deoxygenated hemoglobin. We also note that the responses become noisier for detectors $\mathrm{A}$ and $\mathrm{E}$ as the source-detector separation increases. Detectors B and F show a decrease in activation with increases in the source-detector separation. The results of FD-NIRS analysis are highlighted in the last columns in Figs. 3 and 4. Note that the $y$ axis scale for these plots spans a larger range than for CW-NIRS. In general, FDNIRS analysis yielded hemodynamic changes that were greater than those for CW-NIRS. The strongest (maximum) response was quantified to be $0.31 \mu \mathrm{M}$ in detector $\mathrm{B}$. Hemodynamic responses were quantified as described in Sec. 2.7 (see also Table 3); statistically significant (Wilcoxon signed rank test, testing for response $>0$ ) responses are marked with a "*." 
Table 3 Comparison of change in concentration of oxygenated hemoglobin $\left(\Delta C_{\mathrm{HbO}}\right)$ due to auditory stimulation ( $1000 \mathrm{~Hz}$ pure tone), estimated with CW-NIRS (columns 2 to 5 ) and FD-NIRS (column 6) analysis methods. Magnitude of response was quantified for each subject using methods in Sec. 2.7. Average and standard deviation of the responses across different subjects are displayed here.

\begin{tabular}{|c|c|c|c|c|c|}
\hline \multirow[b]{2}{*}{ Detectors } & \multicolumn{5}{|c|}{$\Delta C_{\mathrm{HbO}}$ in $\mu \mathrm{M}$ for $1000 \mathrm{~Hz}$ pure tone } \\
\hline & Source 1 & Source 2 & Source 3 & Source 4 & FD-NIRS \\
\hline A & $0.08 \pm 0.01$ & $0.1 \pm 0.01$ & $0.1 \pm 0.01$ & $0.06 \pm 0.02^{\mathrm{a}}$ & $0.24 \pm 0.03$ \\
\hline B & $0.13 \pm 0.02$ & $0.11 \pm 0.02$ & $0.12 \pm 0.01$ & $0.11 \pm 0.01$ & $0.28 \pm 0.06$ \\
\hline C & $0.09 \pm 0.02$ & $0.11 \pm 0.02$ & $0.12 \pm 0.02$ & $0.11 \pm 0.02$ & $0.14 \pm 0.03$ \\
\hline D & - & $0.03 \pm 0.01$ & $0.04 \pm 0.01$ & $0.01 \pm 0.01$ & $0.36 \pm 0.02$ \\
\hline$E$ & $0.04 \pm 0.006^{a}$ & $0.04 \pm 0.01^{a}$ & $0.06 \pm 0.01^{\mathrm{a}}$ & $0.06 \pm 0.01^{\mathrm{a}}$ & $0.15 \pm 0.02$ \\
\hline $\mathrm{F}$ & $0.04 \pm 0.01$ & $0.04 \pm 0.01^{a}$ & $0.05 \pm 0.005^{\mathrm{a}}$ & $0.04 \pm 0.005^{\mathrm{a}}$ & $0.04 \pm 0.01$ \\
\hline $\mathrm{G}$ & - & $0.04 \pm 0.02^{a}$ & $0.06 \pm 0.01$ & $0.05 \pm 0.02$ & $0.03 \pm 0.02$ \\
\hline $\mathrm{H}$ & $0.03 \pm 0.01^{a}$ & $0.02 \pm 0.01$ & $0.03 \pm 0.01$ & $0.01 \pm 0.01$ & $0.4 \pm 0.03$ \\
\hline
\end{tabular}

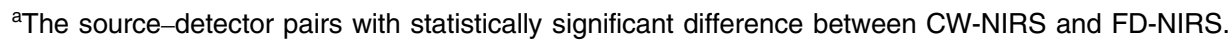

Figures 5 and 6 display the cumulative hemodynamic response curves from CW-NIRS (first four columns) and FD-NIRS (last column) analyses of $70 \mathrm{~dB}$ SPL broadband noise activation, recorded over the posterior and anterior auditory cortices, respectively, from all subjects. Changes in concentrations of oxygenated/deoxygenated hemoglobin are displayed as solid red/blue lines, with corresponding shaded regions denoting 95\% confidence intervals. The period between the dashed vertical lines, i.e., between 0 and $10 \mathrm{~s}$, represents the activation duration. On average, the hemodynamic responses to broadband noise activation were weaker compared with those of pure tone, more diffuse in nature, and stronger in the anterior auditory cortex. This is in line with the tonotopic organization of the auditory cortex..$^{19,21,22}$ With CW-NIRS analysis, the strongest response (maximum value within duration of stimulus presentation) was observed in detectors B and C $(0.12$ and $0.13 \mu \mathrm{M}$, respectively). Hemodynamic responses were quantified as described in Sec. 2.7 (see also Table 4). Statistically significant responses (Wilcoxon signed rank test, testing for response $>0$ ) are marked with a "*” in Figs. 5 and 6. The results of FD-NIRS analysis are highlighted in the last columns in Figs. 5 and 6. Note that the $y$ axis scale for these plots spans a larger range than with CW-NIRS. As with pure tone, FD-NIRS analysis of broadband noise stimulus yielded hemodynamic changes that were greater than those of CW-NIRS. Hemodynamic responses were quantified as described in Sec. 2.7 (see also Table 4); statistically significant (Wilcoxon signed rank test, testing for response $>0$ ) are marked with a "*."

\subsection{Comparison of FD-NIRS and CW-NIRS Hemodynamic Responses to Functional Activation}

Figures 3-6 show that hemodynamic responses to auditory stimulation measured using FD are generally larger than the corresponding CW-NIRS responses. Although both approaches are consistent in identifying the primary activation region, the magnitude of responses is significantly different. Notably, the results from the FD-NIRS analysis show a large range ( 0.35 to $0.41 \mu \mathrm{M}$ ) for the changes in concentration of $\mathrm{HbO}$, whereas in the MBL analysis approach, we observed similar changes but over a smaller range $(0.05$ to $0.16 \mu \mathrm{M})$. We quantified the hemodynamic responses for each detector (FD-NIRS) and each source-detector pair 

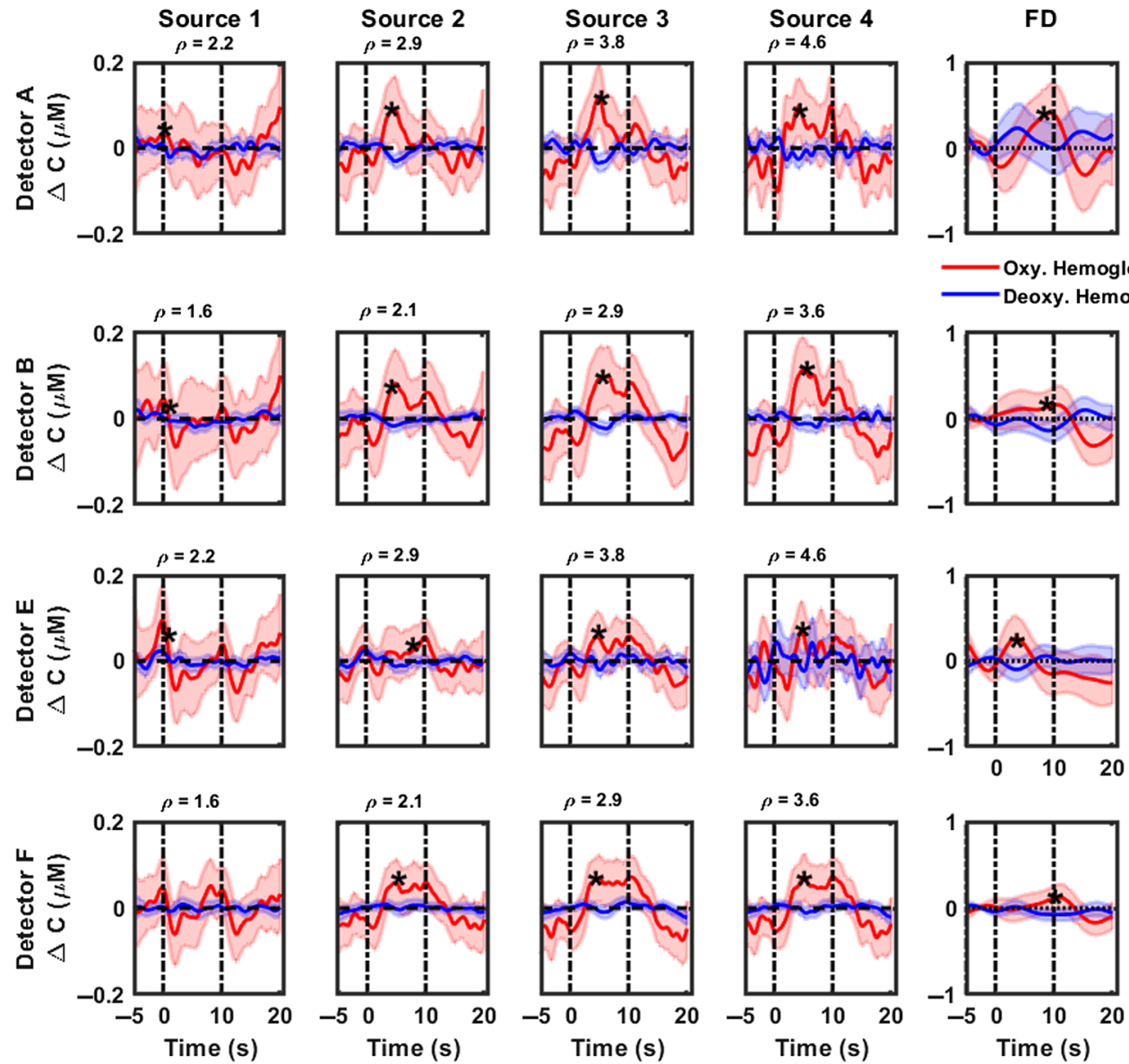

Fig. 5 CW-NIRS and FD-NIRS analyses of functional activation (70 dB SPL broadband noise) recorded over the posterior auditory cortex (detectors $A, B, E$, and F) from all subjects. Rows represent hemodynamic responses recorded at each detector. The first four columns highlight responses recoded at each source position using CW-NIRS (left to right, increasing source-detector separation) and FD-NIRS (last column). In each plot, changes in concentration of oxygenated hemoglobin are plotted in red, and the concentration of deoxyhemoglobin is plotted in blue. The title of each subplot denotes the source-detector separation in $\mathrm{cm}$ for that measurement channel. Note that the $y$ axis scale for FD-NIRS measurements is larger than that of CW-NIRS measurements. Dashed vertical lines indicate the start and end of the stimulus. Shaded regions indicate the variance in the responses. Note that source 1 is closest to and source 4 is farthest from all detectors. Plots marked with a "*" indicate statistically significant responses.

(CW-NIRS) using the methods describes in Sec. 2.7-these are summarized in Table 3 (pure tone stimulus) and Table 4 (broadband noise). On average, FD-NIRS responses were greater than CW-NIRS responses by a factor of 2 . To evaluate if the hemodynamic responses from FD-NIRS were greater than those of CW-NIRS, subject-wise paired Wilcoxon sign rank tests were performed. Briefly, the hemodynamic responses recorded at each detector (FD-NIRS analysis) were compared with the corresponding responses for all source-detector separations (CW-NIRS), paired subject-wise. For pure tone, source 4 in detector A $(\rho=4.6 \mathrm{~cm})$, sources 1 to 4 in detector $\mathrm{E}(\rho=2.2$ to $4.6 \mathrm{~cm})$, sources 2 to 4 in detector $\mathrm{F}(\rho=2.1$ to $3.6 \mathrm{~cm})$, source 2 in detector $\mathrm{G}$ $(\rho=2.9)$, and source 1 in detector $\mathrm{H}(\rho=4.6 \mathrm{~cm})$ were found to be statistically significant. Most of these source-detector pairs correspond to detectors in the posterior auditory cortex. For broadband noise, all sources in detector A $(\rho=2.2$ to $4.6 \mathrm{~cm})$, sources 2 to 4 in detector $\mathrm{E}(\rho=2.9$ to $4.6 \mathrm{~cm})$, all sources in detector $\mathrm{F}(\rho=1.6$ to $3.6 \mathrm{~cm})$, and source 4 in detector $\mathrm{H}$ $(\rho=2.2 \mathrm{~cm})$ were found to be statistically significant. 
Table 4 Comparison of change in concentration of oxygenated hemoglobin $\left(\Delta C_{\mathrm{HbO}}\right)$ due to auditory stimulation (70 dB SPL broadband noise), estimated with CW-NIRS (columns 2 to 5 ) and FDNIRS (column 6) analysis methods. Magnitude of response was quantified for each subject using methods in Sec. 2.7. Average and standard deviation of the responses across different subjects are displayed here.

\begin{tabular}{|c|c|c|c|c|c|}
\hline \multirow[b]{2}{*}{ Detectors } & \multicolumn{5}{|c|}{$\Delta C_{\mathrm{HbO}}$ in $\mu \mathrm{M}$ for broadband noise } \\
\hline & Source 1 & Source 2 & Source 3 & Source 4 & FD-NIRS \\
\hline A & $0.04 \pm 0.01^{a}$ & $0.07 \pm 0.01^{\mathrm{a}}$ & $0.09 \pm 0.02^{\mathrm{a}}$ & $0.07 \pm 0.02^{\mathrm{a}}$ & $0.38 \pm 0.03$ \\
\hline B & $0.07 \pm 0.01$ & $0.09 \pm 0.01$ & $0.12 \pm 0.01$ & $0.12 \pm 0.01$ & $0.15 \pm 0.01$ \\
\hline C & $0.1 \pm 0.01$ & $0.1 \pm 0.01$ & $0.13 \pm 0.01$ & $0.11 \pm 0.01$ & $0.33 \pm 0.02$ \\
\hline D & - & $0.05 \pm 0.01$ & $0.04 \pm 0.01$ & $0.03 \pm 0.008$ & $0.14 \pm 0.01$ \\
\hline $\mathrm{E}$ & $0.05 \pm 0.01$ & $0.05 \pm 0.01^{\mathrm{a}}$ & $0.05 \pm 0.01^{\mathrm{a}}$ & $0.06 \pm 0.01^{a}$ & $0.17 \pm 0.03^{a}$ \\
\hline $\mathrm{F}$ & $0.06 \pm 0.01^{a}$ & $0.05 \pm 0.01^{\mathrm{a}}$ & $0.06 \pm 0.01^{\mathrm{a}}$ & $0.06 \pm 0.01^{a}$ & $0.1 \pm 0.02$ \\
\hline G & - & $0.04 \pm 0.005$ & $0.04 \pm 0.01$ & $0.05 \pm 0.01$ & $0.027 \pm 0.03$ \\
\hline $\mathrm{H}$ & $0.04 \pm 0.01$ & $0.05 \pm 0.01$ & $0.05 \pm 0.01$ & $0.06 \pm 0.02^{\mathrm{a}}$ & $0.023 \pm 0.01$ \\
\hline
\end{tabular}

aThe source-detector pairs with statistically significant difference between CW-NIRS and FD-NIRS.

\subsection{Numerical Simulation of FD-NIRS and CW-NIRS Hemodynamic Responses to Functional Activation}

To validate our results, we used a finite-element-based forward model of tissue light propagation, implemented in NIRFAST, ${ }^{29}$ to simulate functional activation responses that could be analyzed using both CW-NIRS and FD-NIRS approaches. Briefly, a homogenous tissue surface was configured as a $50 \mathrm{~mm} \times 80 \mathrm{~mm} 2 \mathrm{D}$ mesh (over 16k nodes, $0.5 \mathrm{~mm}$ node distance) with optical properties of $C_{\mathrm{HbO}}=0.01 \mathrm{mM}, C_{\mathrm{HbR}}=0.01 \mathrm{mM}$, and both scattering amplitude and scattering power equal to 1 . In seven different simulations, circular anomalies (simulating focal activation) of different radii $(8,10$, and $12 \mathrm{~mm})$ were placed at multiple depths below the tissue surface $(15,10$, and $8 \mathrm{~mm})$ as shown in Fig. 7 . The size and depths of the anomaly were selected to mimic activation in different sections of the auditory cortex.

Optical properties of the anomaly were set to of $C_{\mathrm{HbO}}=0.06 \mathrm{mM}, C_{\mathrm{HbR}}=$ $0.005 \mathrm{mM},{ }^{7,32,34,41}$ scattering amplitude of 1.34 , and scattering power of 0.56 . NIRFAST was used to solve the forward model of frequency domain photon migration (100 MHz modulation frequency, wavelengths of 690 and $830 \mathrm{~nm}$ ) to record the amplitude and phase of the photon fluence rate at source-detector separations of 10,15, 20, and $30 \mathrm{~mm}$. Simulated amplitude and phase measurements were analyzed using the FD-NIRS approach, whereas amplitude was analyzed with the CW-NIRS approach to estimate the change (from baseline) in concentration of oxygenated and deoxygenated hemoglobin. The \% error in hemodynamic estimates were calculated as

$$
\% \text { error }=100 \times \frac{\left(\Delta C_{\mathrm{HbO} / R}^{\text {measured }}-\Delta C_{\mathrm{HbO} / R}^{\text {simulated }}\right)}{\Delta C_{\mathrm{HbO} / R}^{\text {simulate }}},
$$

where $\Delta C_{\mathrm{HbO}}^{\text {simulated }}=50 \mu \mathrm{M}$ and $\Delta C_{\mathrm{HbR}}^{\text {simulated }}=-0.05 \mu \mathrm{M}$.

Figure 8 summarizes the average error in estimation of hemodynamic changes [Fig. 8(a): \% error in $\Delta C_{\mathrm{HbO}}$ estimates and Fig. 8(b): \% error in $\Delta C_{\mathrm{HbR}}$ estimates], for anomalies of different sizes (each column) and at different depths ( $x$ axis of each plot). In each plot, the red diamonds represent results from multi-distance FD NIRS, blue dots represent results from CW-NIRS (best source-detector separation, i.e., source-detector separation that yielded highest response), and magenta crosses represent results from CW-NIRS (average of all source-detector separations). 

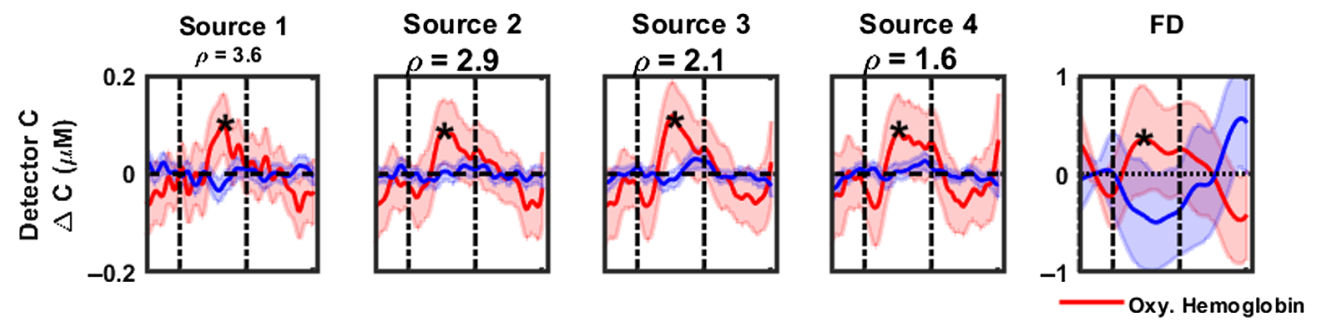

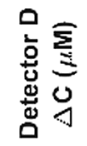
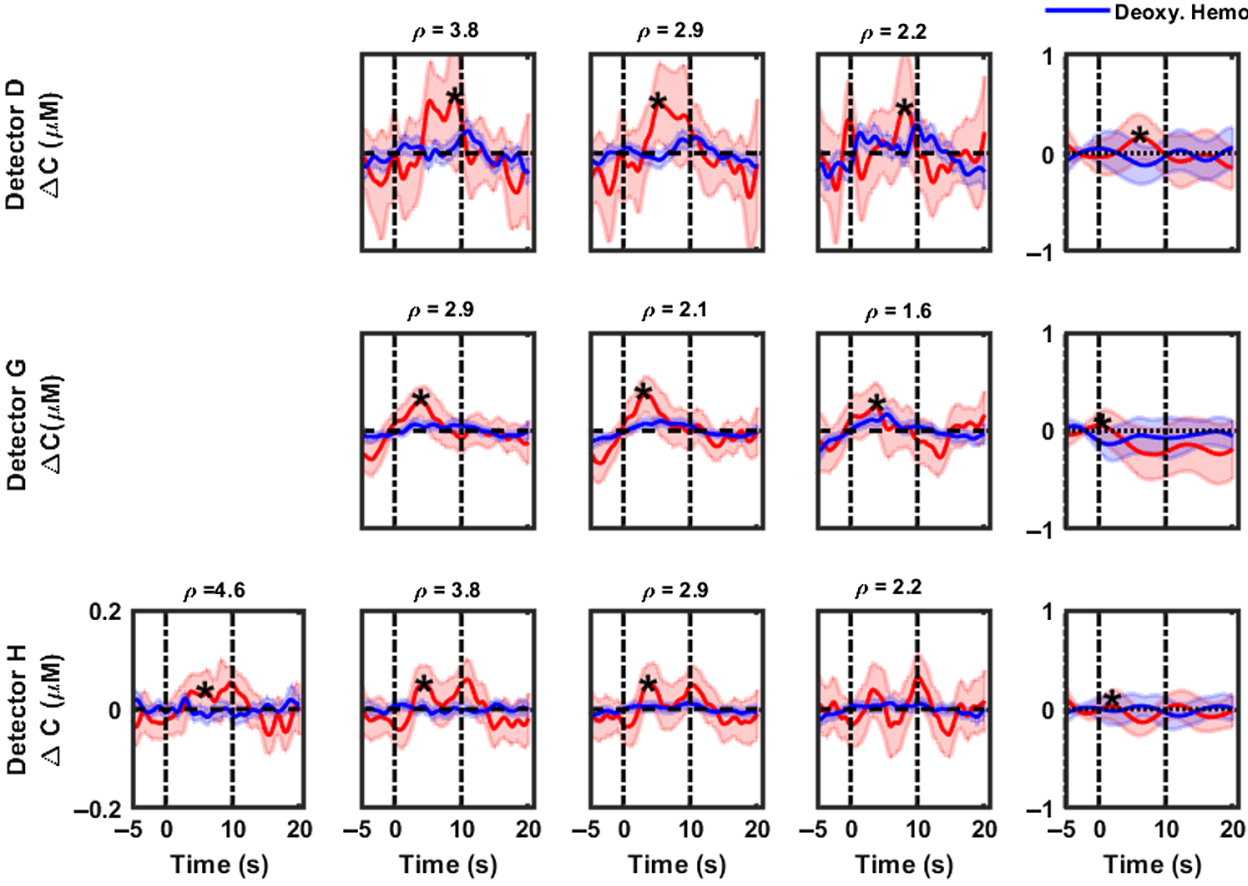

Fig. 6 CW-NIRS and FD-NIRS analyses of functional activation (70 dB SPL broadband noise) recorded over the anterior auditory cortex (detectors $\mathrm{C}, \mathrm{D}, \mathrm{G}$, and $\mathrm{H}$ ) from all subjects. Rows represent hemodynamic responses recorded at each detector. The first four columns highlight responses recoded at each source position using CW-NIRS (left to right, decreasing sourcedetector separation) and FD-NIRS (last column). In each plot, changes in concentration of oxygenated hemoglobin are plotted in red, and the concentration of deoxyhemoglobin is plotted in blue. The title of each subplot denotes the source-detector separation in $\mathrm{cm}$ for that measurement channel. Note that the $y$ axis scale for FD-NIRS measurements is larger than that of CW-NIRS measurements. Dashed vertical lines indicate the start and end of the stimulus. Shaded regions indicate the variance in the responses. Note that source 4 is closest to and source 1 is farthest from all detectors. Detector $D$ and $G$ are not included here because they were too noisy. Plots marked with a "*” indicate statistically significant responses.

We can observe that the error in estimated changes of the concentrations increase with anomaly size and depth in FD results. Anomalies that were at shallow depths likely had uniform sampling of the perturbation by all source-detector separations, lessening the impact of the partial volume effect. By contrast, the concentration changes estimated with MBL law were relatively constant. Note that, although the error in estimated concentration changes is lower with CW-NIRS, the overall magnitude is still high. This could be because the presence of the anomalies could violate the inherent assumption of homogenous media in both MBL and FD-NIRS analyses. With FDNIRS, our results show that the use of multiple source-detector separations in the analysis contributes to errors to a larger extent.

\section{Discussion}

The availability of commercial fNIRS devices, the standardization of data processing methods, ${ }^{47}$ and the development of algorithms to correct for motion artifacts ${ }^{48-52}$ or extra-cerebral 

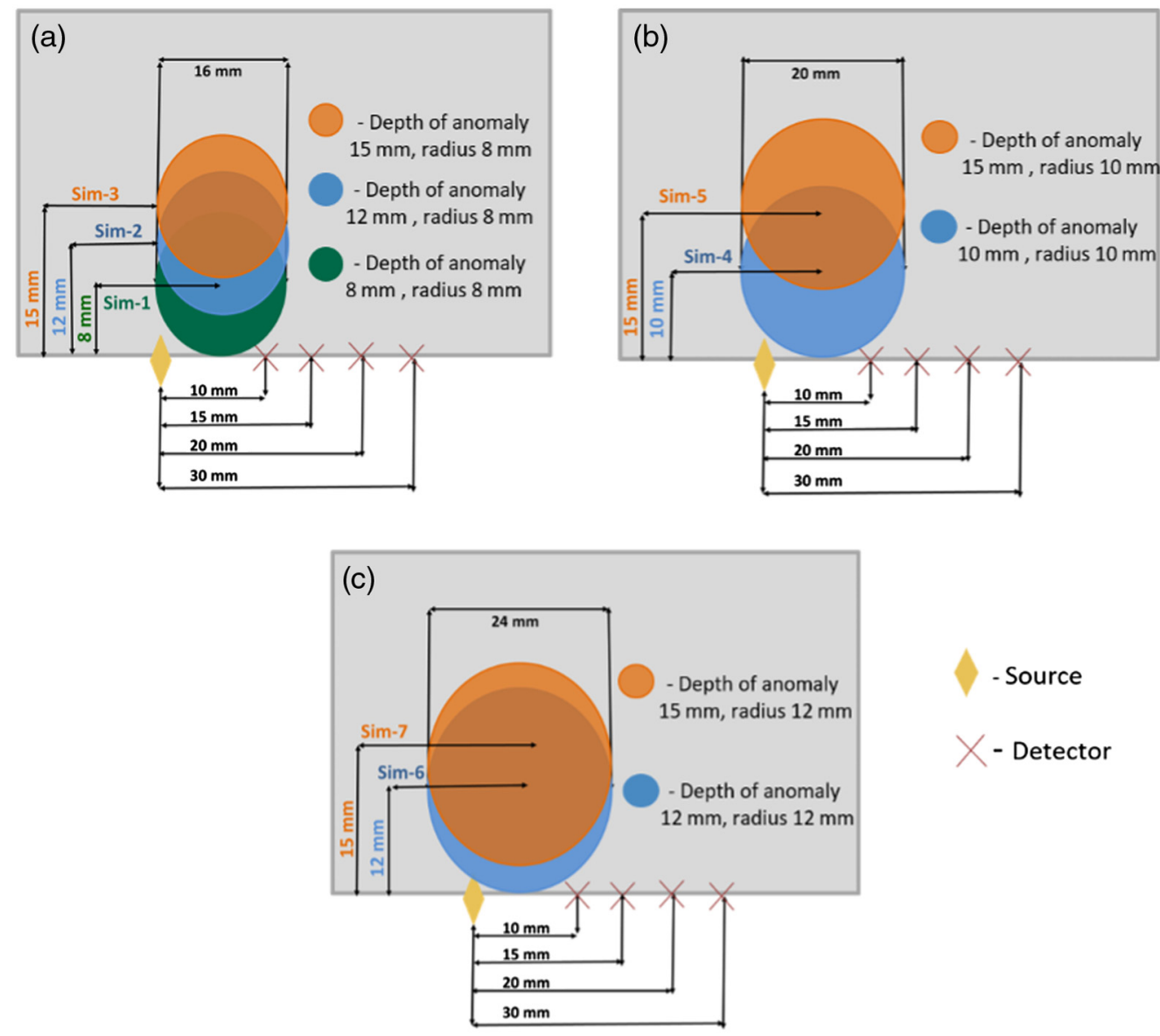

Fig. 7 Schematic of homogenous slab geometry used for finite-element (NIRFAST) simulations. Yellow rhombus indicates of the position of the detector, and crosses indicate the positions of four sources, with source-detector separations ranging from 1 to $3 \mathrm{~cm}$. The colored circles highlight the position of circular anomalies that simulates the region with functional activation. The background properties for tissues were $C_{\mathrm{HbO}}=0.01 \mathrm{mM}, C_{\mathrm{HbR}}=0.01 \mathrm{mM}$, scattering amplitude $=1$, and scattering power $=1$. The optical properties of the anomaly were $C_{\mathrm{HbO}}=0.06 \mathrm{mM}$, $C_{\mathrm{HbR}}=0.005 \mathrm{mM}$, scattering amplitude $=1.34$, and scattering power $=0.56$.

hemodynamic changes ${ }^{53,54}$ have helped expand functional activation measurements to challenging experiments such as auditory stimulation - in which the activation regions are expected to be 2 to $3 \mathrm{~cm}$ below the surface of the head. However, most functional activation experiments-with some exceptions ${ }^{26,55}$ - still use $\mathrm{CW}$ approaches despite the availability of commercial instruments that can implement the frequency domain approach. Indeed, this is the first study to use a frequency domain approach to record hemodynamic changes due to functional activation of the auditory cortex. Our primary objective was to compare the more popular $\mathrm{CW}$ approach with the more rigorous FD approach.

Using the CW-NIRS approach, we measured a $0.1 \approx 0.2 \mu \mathrm{M}$ change in concentration of oxygenated hemoglobin, which is consistent with similar $\mathrm{CW}$ measurements in the literature. ${ }^{7,18,32}$ For example, Chen et al., ${ }^{7}$ Issa et al., ${ }^{18}$ and Wiggins et al ${ }^{32}$ observed a maximum $C_{\mathrm{HbO}}$ change of $0.4,0.1$, and $0.2 \mu \mathrm{M}$, respectively. With the FD-NIRS approach, however, we measured a consistently higher change in concentration of oxygenated hemoglobin. Both CW and frequency domain results were obtained from the same data set, albeit using different models of photon diffusion to account for tissue scattering effects. The $\mathrm{CW}$ approach analyzes intensity measurements at each source-detector separation and approximates scattering effects with the MBL law. The FD approach directly measures the tissue scattering coefficient $\left(\mu_{s}^{\prime}\right)$ with photon fluence measurements at multiple source-detector separations. The multi-distance approach inherently assumes that measurements made at different source-detector separations are equally sensitive to 
(a) Radius of anomaly $=8 \mathrm{~mm}$

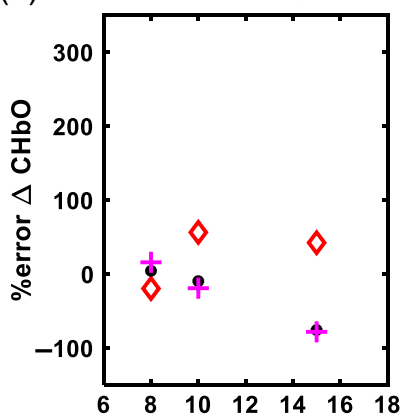

(b) Radius of anomaly $=8 \mathrm{~mm}$

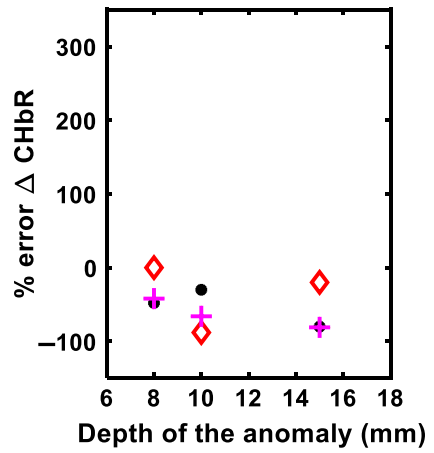

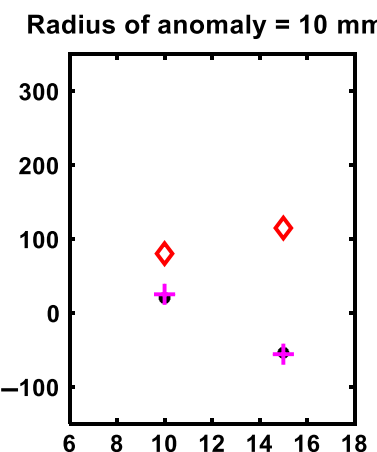
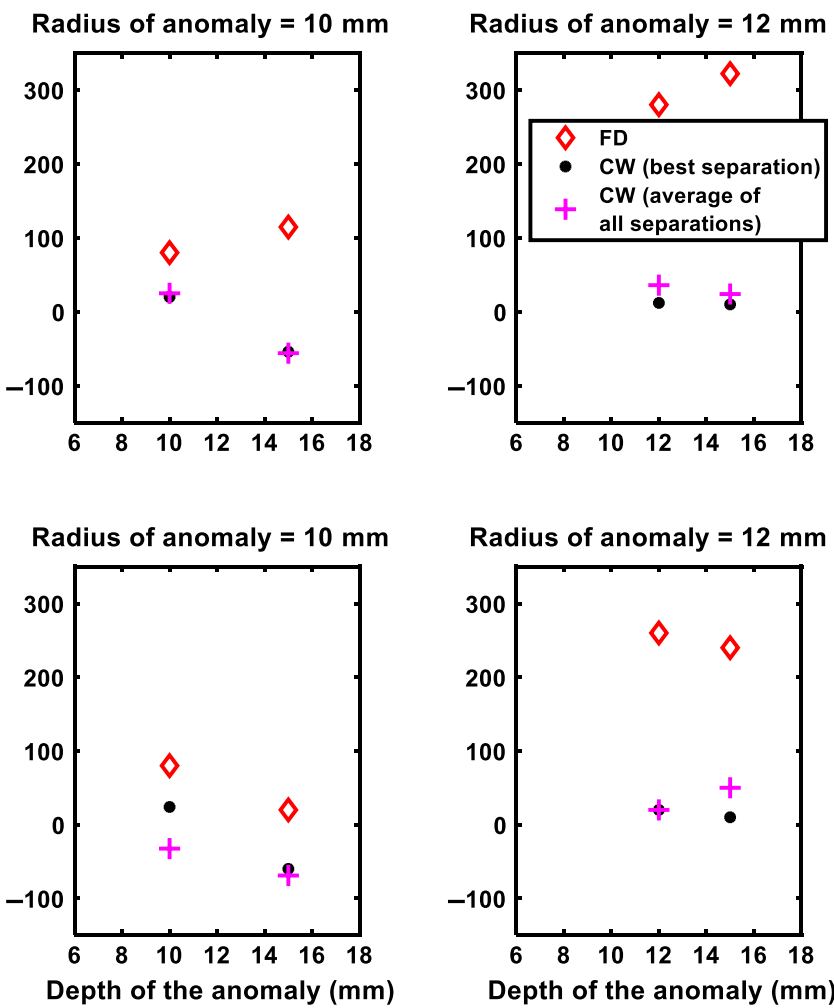

Fig. 8 Results of seven finite-element simulations of function activation showing \% error in estimations of (a) oxygenated and (b) deoxygenated hemoglobin. Red diamonds represent results from multi-distance FD NIRS, blue dots represent results from CW-NIRS (best source-detector separation, i.e., source-detector separation that yielded highest response), and magenta crosses represent results from CW-NIRS (average of all source-detector separations).

changes in tissue optical properties - this assumption is often invalidated in functional activation measurements that feature focal hemodynamic changes. In this study, multi-distance FD measurements of the photon fluence rate were performed at source-detector separations of 1.6 to $4.5 \mathrm{~cm}$; longer separations are sensitive to deeper tissue depths. Thus a focal change in tissue absorption at say 10 to $15 \mathrm{~mm}$ below the surface will elicit a greater change in photon fluence rates at $2.5 \mathrm{~cm}$ source-detector separation than $1.5 \mathrm{~cm}$. This partial volume effect could explain why the FD-NIRS analysis systematically overestimates hemodynamic response to functional activation. To verify our results, we performed controlled finite-element simulations of the functional activation process. Our simulation results confirm that, due to partial volume effects, heterogeneous/focal tissue oxygenation changes, such as those due to functional activation, are better measured with single-distance rather than multi-distance approaches.

We note a few limitations of our comparison. Despite our results suggesting that FD-NIRS approaches can overestimate functional activation hemodynamics, frequency domain instruments remain superior to simpler CW-NIRS instruments because they can directly measure scattering effects. One limitation of this study is that it compared CW-NIRS with multi-distance FD approaches. Some or all of the biases introduced by partial volume effects could potentially be addressed by newer frequency domain instruments that operate at a single source-detector separation $^{56,57}$ or fast look-up table inversion methods for optical property estimation. ${ }^{3}$ These approaches typically solve for the photon diffusion equation at multiple modulation frequencies, which are less susceptible to partial volume effects. Comparison of single-distance CW-NIRS with single-distance FD-NIRS is an important future study. A second limitation is the use of homogeneous tissue models for the analysis of functional activation hemodynamics. Extra cerebral flow/oxygenation changes can significantly confound functional activation measurements. ${ }^{53}$ Here we have partially mitigated these effects by only using measurements at a longer source-detector separation for FD-NIRS analysis. A better approach would be to 
use multi-layered tissue diffusion models. ${ }^{58-60}$ Another potential solution to address the partial volume effects is to use time-domain approaches ${ }^{61}$ that use optical measurements from one source-detector pair to fully solve the photon diffusion equation. However, because they need specialized light sources and complex instrumentation, TD instruments are not commercially available and are less common in the clinic. A more practical alternative is diffuse optical tomography, which uses multiple single-distance $\mathrm{CW}$ diffuse optical measurements to create a 2D/3D map of tissue oxygenation changes. Notably, Culver et al. ${ }^{62}$ showed that accurate determination of functional activation changes can be achieved by reconstructing an image of tissue hemodynamic changes from a plurality of single-distance $\mathrm{CW}$ diffuse optical measurements.

\section{Conclusion}

Functional stimulation of the auditory cortex was studied using CW-NIRS and FD-NIRS approaches and their results were compared. The responses from multi-distance FD-NIRS yielded baseline measurements, whereas the CW-NIRS approach estimated the changes in the concentration of $\mathrm{HbO}$ and $\mathrm{HbR}$. For functional activation changes, i.e., focal changes, $\mathrm{CW}-\mathrm{NIRS}$ offered better estimation of changes in concentration of $\mathrm{HbO}$ and $\mathrm{HbR}$, compared with that of the multi-distance FD-NIRS analysis. The results obtained suggest that the single-distance MBL analysis approach is well suited for measuring changes due to functional activation stimulations.

\section{Disclosures}

The authors have no conflicts of interest.

\section{Acknowledgments}

This work was partially supported by the National Institutes of Health (No. R21GM137209, A. B. P.), USF College of Engineering (A. B. P.), and USF College of Behavioral and Community Sciences (A. C. E.).

\section{References}

1. A. Villringer, "Non-invasive optical spectroscopy and imaging of human brain function," Trends Neurosci. 20(10), 435-442 (1997).

2. Y. Hoshi and M. Tamura, "Dynamic multichannel near-infrared optical imaging of human brain activity," J. Appl. Physiol. 75(4), 1842-1846 (1993).

3. M. B. Applegate, C. A. Gómez, and D. M. Roblyer, "Modulation frequency selection and efficient look-up table inversion for frequency domain diffuse optical spectroscopy," J. Biomed. Opt. 26(3), 036007 (2021).

4. H. Obrig et al., "Continuous spectrum near-infrared spectroscopy approach in functional activation studies in the human adult," Proc. SPIE 2926, 58-66 (1996).

5. M. Ferrari and V. Quaresima, "A brief review on the history of human functional nearinfrared spectroscopy (fNIRS) development and fields of application," NeuroImage 63(2), 921-935 (2012).

6. T. Wilcox et al., "Using near-infrared spectroscopy to assess neural activation during object processing in infants," J. Biomed. Opt. 10(1), 011010 (2005).

7. L.-C. Chen et al., "Association of concurrent fNIRS and EEG signatures in response to auditory and visual stimuli," Brain Topogr. 28(5), 710-725 (2015).

8. C.-M. Lu et al., "Use of fNIRS to assess resting state functional connectivity," J. Neurosci. Methods 186(2), 242-249 (2010).

9. D. R. Leff et al., "Assessment of the cerebral cortex during motor task behaviours in adults: a systematic review of functional near infrared spectroscopy (fNIRS) studies," NeuroImage 54(4), 2922-2936 (2011). 
10. N. H. Kashou et al., "Hand-grasping and finger tapping induced similar functional nearinfrared spectroscopy cortical responses," Neurophotonics 3(2), 025006 (2016).

11. M. Carrieri et al., "Prefrontal cortex activation upon a demanding virtual hand-controlled task: a new frontier for neuroergonomics," Front. Hum. Neurosci. 10, 53 (2016).

12. K.-S. Hong, N. Naseer, and Y.-H. Kim, "Classification of prefrontal and motor cortex signals for three-class fNIRS-BCI," Neurosci. Lett. 587, 87-92 (2015).

13. N. Naseer and K.-S. Hong, "fNIRS-based brain-computer interfaces: a review," Front. Hum. Neurosci. 9, 3 (2015).

14. R. Zimmermann et al., "Towards a BCI for sensorimotor training: initial results from simultaneous fNIRS and biosignal recordings," in Annu. Int. Conf. IEEE Eng. Med. and Biol. Soc., IEEE (2011).

15. P. P. S. Mohammad, "Quantitative measurement of cerebral hemodynamics during activation of auditory cortex with single-and multi-distance near infrared spectroscopy," Master's Thesis, University of South Florida, Tampa, FL (2018).

16. K.-S. Hong and H. Santosa, "Decoding four different sound-categories in the auditory cortex using functional near-infrared spectroscopy," Hear. Res. 333, 157-166 (2016).

17. M. J. Khan and K.-S. Hong, "Hybrid EEG-fNIRS-based eight-command decoding for BCI: application to quadcopter control," Front. Neurorobot. 11, 1 (2017).

18. M. Issa et al., "Human auditory and adjacent nonauditory cerebral cortices are hypermetabolic in tinnitus as measured by functional near-infrared spectroscopy (fNIRS)," Neural Plast. 2016, 1-13 (2016).

19. M. Moerel, F. De Martino, and E. Formisano, "An anatomical and functional topography of human auditory cortical areas," Front. Neurosci. 8, 225 (2014).

20. J. Rademacher et al., "Probabilistic mapping and volume measurement of human primary auditory cortex," Neuroimage 13(4), 669-683 (2001).

21. C. Humphries, E. Liebenthal, and J. R. J. N. Binder, "Tonotopic organization of human auditory cortex," Neuroimage 50(3), 1202-1211 (2010).

22. T. M. Talavage et al., "Tonotopic organization in human auditory cortex revealed by progressions of frequency sensitivity," J. Neurophysiol. 91(3), 1282-1296 (2004).

23. A. Duncan et al., "Optical pathlength measurements on adult head, calf and forearm and the head of the newborn infant using phase resolved optical spectroscopy," Phys. Med. Biol. 40(2), 295 (1995).

24. A. Duncan et al., "Multiwavelength, wideband, intensity-modulated optical spectrometer for near-infrared spectroscopy and imaging," Proc. SPIE 1888, 248-257 (1993).

25. M. Calderon-Arnulphi et al., "Detection of cerebral ischemia in neurovascular surgery using quantitative frequency-domain near-infrared spectroscopy," J. Neurosurg. 106(2), 283-290 (2007).

26. D. J. Davies et al., "Frequency-domain vs continuous-wave near-infrared spectroscopy devices: a comparison of clinically viable monitors in controlled hypoxia," J. Clin. Monitor. Comp. 31(5), 967-974 (2017).

27. S. Fantini et al., "Non-invasive optical monitoring of the newborn piglet brain using continuous-wave and frequency-domain spectroscopy," Phys. Med. Biol. 44(6), 1543-1563 (1999).

28. H. Y. Ma et al., "Quantitative study of hypoxia stress in piglet brain by IQ phase modulation oximetry," Proc. SPIE 3597, 642-649 (1999).

29. H. Dehghani et al., "Near infrared optical tomography using NIRFAST: algorithm for numerical model and image reconstruction," Commun. Numer. Methods Eng. 25(6), 711-732 (2008).

30. S. Fantini et al., "Quantitative determination of the absorption spectra of chromophores in strongly scattering media: a light-emitting-diode based technique," Appl. Opt. 33(22), 52045213 (1994).

31. M. Schecklmann et al., "Functional near-infrared spectroscopy to probe state- and trait-like conditions in chronic tinnitus: a proof-of-principle study," Neural Plast. 2014, 1-8 (2014).

32. I. M. Wiggins et al., "Speech-evoked activation in adult temporal cortex measured using functional near-infrared spectroscopy (fNIRS): are the measurements reliable?" Hear. Res. 339, 142-154 (2016). 
33. S.-H. Yoo et al., "Decoding multiple sound-categories in the auditory cortex by neural networks: an fNIRS study," Front. Hum. Neurosci. 15, 636191 (2021).

34. F. Putze et al., "Hybrid fNIRS-EEG based classification of auditory and visual perception processes," Front. Neurosci. 8, 373 (2014).

35. S. A. F. Sergio, "Frequency-domain techniques for cerebral and functional near-infrared spectroscopy," Front. Neurosci. 14, 300 (2020).

36. S. Wojtkiewicz, T. Durduran, and H. Dehghani, "Time-resolved near infrared light propagation using frequency domain superposition," Biomed. Opt. Express 9(1), 41-54 (2018).

37. T. Durduran et al., "Diffuse optics for tissue monitoring and tomography," Rep. Prog. Phys. 73(7), 076701 (2010).

38. S. Fantini, "Semi-infinite-geometry boundary problem for light migration in highly scattering media: a frequency-domain study in the diffusion approximation," J. Opt. Soc. Am. B 11(10), 2128-2138 (1994).

39. W. B. Baker, "Optical cerebral blood flow monitoring of mice to men," PhD Dissertation, University of Pennsylvania, Philadelphia, PA (2015).

40. E. M. Buckley, "Cerebral hemodynamics in high-risk neonates probed by diffuse optical spectroscopies," PhD dissertation, University of Pennsylvania, Philadelphia, PA (2011).

41. N. Curry et al., "Direct determination of diffusion properties of random media from speckle contrast," Opt. Lett. 36(17), 3332-3334 (2011).

42. S. Matcher et al., "Use of the water absorption spectrum to quantify tissue chromophore concentration changes in near-infrared spectroscopy," Phys. Med. Biol. 39(1), 177 (1994).

43. A. Demel et al., "Effect of different assumptions for brain water content on absolute measures of cerebral oxygenation determined by frequency-domain near-infrared spectroscopy in preterm infants: an observational study," BMC Pediatr. 14(1), 1-6 (2014).

44. J. Pivarnik et al., Nutrition in Exercise and Sport, CRC Press, Boca Raton, FL (1994).

45. L. Gagnon et al., "Quantification of the cortical contribution to the NIRS signal over the motor cortex using concurrent NIRS-fMRI measurements," BMC Pediatr. 59(4), 39333940 (2012).

46. G. Strangman et al., "A quantitative comparison of simultaneous BOLD fMRI and NIRS recordings during functional brain activation," Neuroimage 17(2), 719-731 (2002).

47. S. L. Novi et al., "Functional near-infrared spectroscopy for speech protocols: characterization of motion artifacts and guidelines for improving data analysis," Neurophotonics 7(1), 015001 (2020).

48. J. W. Barker, A. Aarabi, and T. J. Huppert, "Autoregressive model based algorithm for correcting motion and serially correlated errors in fNIRS," Biomed. Opt. Express 4(8), 1366-1379 (2013).

49. J. W. Barker et al., "Correction of motion artifacts and serial correlations for real-time functional near-infrared spectroscopy," Neurophotonics 3(3), 031410 (2016).

50. S. Brigadoi et al., "Motion artifacts in functional near-infrared spectroscopy: a comparison of motion correction techniques applied to real cognitive data," NeuroImage 85(Pt 1 (0 1)), 181-191 (2014).

51. J. J. Selb et al., "Effect of motion artifacts and their correction on near-infrared spectroscopy oscillation data: a study in healthy subjects and stroke patients," J. Biomed. Opt. 20(5), 056011 (2015).

52. M. A. Yucel et al., "Targeted principle component analysis: a new motion artifact correction approach for near-infrared spectroscopy," J. Innov. Opt. Health Sci. 7(2), 1350066 (2014).

53. W. B. Baker et al., "Pressure modulation algorithm to separate cerebral hemodynamic signals from extracerebral artifacts," Neurophotonics 2(3), 035004 (2015).

54. G. Bauernfeind et al., "Separating heart and brain: on the reduction of physiological noise from multichannel functional near-infrared spectroscopy (fNIRS) signals," J. Neural Eng. 11(5), 056010 (2014).

55. G. Zhang et al., "Brain perfusion monitoring with frequency-domain and continuous-wave near-infrared spectroscopy: a cross-correlation study in newborn piglets," Phys. Med. Biol. 45(11), 3143-3158 (2000). 
56. A. Torjesen, R. Istfan, and D. Roblyer, "Ultrafast wavelength multiplexed broad bandwidth digital diffuse optical spectroscopy for in vivo extraction of tissue optical properties," J. Biomed. Opt. 22(3), 036009 (2017).

57. A. Sassaroli, G. Blaney, and S. Fantini, "Dual-slope method for enhanced depth sensitivity in diffuse optical spectroscopy," J. Opt. Soc. Am. A 36(10), 1743-1761 (2019).

58. R. K. Almajidy et al., "Dual layered models of light scattering in the near infrared a: optical measurements and simulation," in 41st Annu. Int. Conf. IEEE Eng. Med. and Biol. Soc., IEEE (2019).

59. B. Hallacoglu, A. Sassaroli, and S. Fantini, "Optical characterization of two-layered turbid media for non-invasive, absolute oximetry in cerebral and extracerebral tissue," PLoS One 8(5), e64095 (2013).

60. H. Ding et al., "Optical topography guided semi-three-dimensional diffuse optical tomography for a multi-layer model of occipital cortex: a pilot methodological study," Proc. SPIE 9690, 96901S (2016).

61. J. Sutin et al., "Time-domain diffuse correlation spectroscopy," Optica 3(9), 1006-1013 (2016).

62. J. P. Culver et al., "Diffuse optical tomography for mapping human brain function," in IEEE/ NLM Life Sci. Syst. and Appl. Workshop, IEEE (2006).

Penaz Parveen Sultana Mohammad is a $\mathrm{PhD}$ student in the Department of Electrical Engineering at University of South Florida. She received her BE in instrument technology from Andhra University, Visakhapatnam, India. Her research focuses on modeling and analysis of functional brain activity measured using fNIRS and DCS.

Sittiprapa Isarangura, $\mathrm{PhD}$ is an instructor at the Department of Communication Sciences and Disorders, Mahidol University, and a courtesy faculty at the Department of Communication Sciences and Disorders, University of South Florida. Her research emphasizes psychoacoustic and human physiology. She mainly focuses on understanding how the human auditory system processes complex sounds and how humans use available auditory cues. She also provides audiological service for both adult and pediatric patients.

Ann Eddins, PhD, MBA, CCC-A, is professor in the Department of Communication Sciences \& Disorders at the University of South Florida and co-director of the Auditory \& Speech Sciences Laboratory. She is a neuroscientist and clinical audiologist with research and clinical interests aimed at understanding the neural correlates of auditory perception and neural plasticity with aging, hearing loss, tinnitus, and rehabilitative intervention. Her research has been supported by NSF, NIH, industry, and private foundations.

Ashwin B. Parthasarathy, $\mathrm{PhD}$, is an assistant professor in the Department of Electrical Engineering at the University of South Florida where he leads the Translational Optics Imaging and Spectroscopy Laboratory. His research interests are in the development of optical technology for clinical biomedical applications, focusing on the imaging and monitoring of cerebral blood flow with laser speckle contrast imaging and diffuse correlation spectroscopy, respectively. 